$11-6-2016$

\title{
Relationships between digit ratio (2D:4D) and basketball performance in Australian men
}

\author{
Nathan A. Frick \\ Melissa J. Hull \\ John T. Manning \\ Grant Tomkinson \\ University of North Dakota, grant.tomkinson@und.edu
}

\section{How does access to this work benefit you? Let us know!}

Follow this and additional works at: https://commons.und.edu/ehb-fac

Part of the Health and Physical Education Commons

\section{Recommended Citation}

Nathan A. Frick, Melissa J. Hull, John T. Manning, et al.. "Relationships between digit ratio (2D:4D) and basketball performance in Australian men" (2016). Education, Health \& Behavior Studies Faculty Publications. 21.

https://commons.und.edu/ehb-fac/21

This Article is brought to you for free and open access by the Department of Education, Health \& Behavior Studies at UND Scholarly Commons. It has been accepted for inclusion in Education, Health \& Behavior Studies Faculty Publications by an authorized administrator of UND Scholarly Commons. For more information, please contact und.commons@library.und.edu. 


\section{Relationships between digit ratio (2D:4D) and basketball performance in Australian men}

Nathan A. Frick, Melissa J. Hull, John T. Manning, Grant R. Tomkinson

\section{Abstract \\ Objective}

To investigate relationships between the digit ratio (2D:4D) and competitive basketball performance in Australian men.

\section{Methods}

Using an observational cross-sectional design a total of 221 Australian basketball players who competed in the Olympic Games, International Basketball Federation World Championships/Cup, Australian National Basketball League, Central Australian Basketball League or socially had their 2D:4Ds measured. Analysis of variance was used to assess differences in mean 2D:4Ds between men playing at different competitive standards, with relationships between 2D:4Ds and basketball game-related statistics assessed using Pearson's product moment correlations in men playing at a single competitive standard.

\section{Results}

There were significant differences between competitive standards for the left 2D:4D following Bonferroni correction, but not for the right 2D:4D, with basketballers who achieved higher competitive standards tending to have lower left 2D:4Ds. No important correlations between 2D:4D and basketball game-related statistics were found, with correlations typically negligible.

\section{Conclusions}

This study indicated that the 2D:4D can discriminate between basketballers competing at different standards, but not between basketballers within a single competitive standard using objective gamerelated statistics.

\section{Introduction}

Digit ratio (2D:4D), or the ratio of the length of the second digit ("index finger") and the length of the fourth digit ("ring finger"), is a nonfunctional trait that has been substantially and negatively correlated with behavior, fertility, health, and sporting ability (Manning, 2002). Digit ratio tends to be lower (i.e., the fourth digit is relatively longer than the second digit) in males than in females and similar in both the right and left hands (Hönekopp and Schuster, 2010; Manning, 2002). Substantial differences in 2D:4D have also been observed between different ethnic groups, for example people of African descent typically have lower 2D:4Ds compared to Caucasians (Manning et al., 2007).

Research suggests the relative lengths of the digits are typically set by week 13 of fetal development (Malas et al., 2006) and remain relatively stable across the lifespan (McIntyre et al., 2005; Trivers et al., 2006). The sexual dimorphism of 2D:4D is thought to be due to differential exposure to sex steroids (testosterone \& estrogen) as the fetus grows (Manning, 2002; Zheng and Cohn, 2011). Recent evidence has shown that prenatal testosterone and estrogen influence the 2D:4D by activating/deactivating a list of skeletogenic genes that also have influences on development of the nervous system and important disease predispositions (Zheng and Cohn, 2011). 
Prenatal testosterone has been linked to a number of extra-genital effects on the growing fetus. These include increased skeletal development, increased growth, and development of the brain and several physiological systems including urogenital and cardiovascular systems (Kimura, 1996; Manning and Hill, 2009; Mortlock and Innis, 1997). Early evidence suggested that increased prenatal testosterone exposure is responsible for increased growth and development in the right hemisphere of the brain, as well as increased strength and efficiency of the cardiovascular system (Geschwind and Galaburda, 1987). There is also some evidence suggesting that individuals with lower 2D:4Ds may have enhanced visualspatial processing abilities, which are important for sports/physical performance (Manning and Taylor, 2001; Peters et al., 2007; Tlauka et al., 2008).

There is mounting evidence substantially linking 2D:4D with sporting performance, which has shown that individuals with lower 2D:4Ds tend to perform better athletically. In a meta-analysis of 25 studies, Hönekopp and Schuster (2010) reported that the overall relationship between 2D:4D and sporting performance was negative and small $(r=-0.26, P<0.001)$ indicating that athletes with a lower $2 \mathrm{D}: 4 \mathrm{D}$ perform better athletically. Many of the studies reviewed by Hönekopp and Schuster (2010) examined the 2D:4D-performance link in individual sports, with subsequent studies linking 2D:4D to team sports performance showing similar relationships (Panda et al., 2014; Schorer et al., 2013; Sudhakar et al., 2014). Of these studies, nearly all of the attention has been on field-based team sports performance (e.g., soccer and rugby), with little known about the links between 2D:4D and court-based team sports performance. Whilst not examined by Hönekopp and Schuster (2010), differences should also be considered between open-skill sports (i.e., unstable, unpredictable, externally paced environments where the athlete is required to react to a stimulus) and closed-skill sports (i.e., stable, predictable, self-paced environments where the athlete initiates the action; Wang et al., 2013). Because of differences in sporting demands, athletes who participate in open-skilled sports probably develop greater flexibility in visual attention, decision making, and action execution relative to athletes participating in closed-skill sports (Taddei et al., 2012). Two meta-analyses have supported this concept, finding that athletes who participate in open-skill sports perform better in cognitively demanding tasks compared to closed-skill athletes (Mann et al., 2007; Voss et al., 2010). Thus it is interesting to consider if the 2D:4D has a stronger relationship with performance in open-skill as opposed to closed-skill athletes or with team versus individual athletes. Unfortunately, a paucity of research investigating the relationship between 2D:4D and open-skilled team sports performance limits our knowledge and makes comparisons difficult.

There is very limited evidence examining the specific relationship between 2D:4D and basketball performance. While Tester and Campbell (2007) were the first to examine 2D:4Ds in basketball players, they presented pooled findings across a number of sports rather than separate results for individual sports. The aim of this study therefore is to quantify the relationships between 2D:4D and competitive basketball performance in Australian men. Specifically this study examined the differences in mean 2D:4Ds between Australian men playing basketball at different competitive standards, as well as the relationships between 2D:4Ds and basketball game-related statistics in Australian men playing at the same competitive standard.

\section{Methods}

\subsection{Participants}

A total of 249 Australian male basketball players (aged 18-40 years) who played across a range of competitive standards volunteered for this study. Informed written consent was obtained from all participants. Because of reported differences in 2D:4Ds across different ethnicities (Manning et al., 2007), only participant data for men of European descent (representing 94\% or 234/249) were retained for final analysis. Participant data were also excluded on the basis of poor image quality (i.e., one or more digit 
landmarks could not be confidently identified from the digital images; $n=3$ ) and if the participant had experienced a substantial injury (e.g., sprain, dislocation or break) to either their second or fourth digits of either hand $(n=10)$. The final sample size comprised 221 Australian men who played at one of the four competitive standards in basketball (at the senior level): International: Olympic Games and/or International Basketball Federation (FIBA) World Championships/Cup for Australia; National: Australian National Basketball League (NBL); State: Central Australian Basketball League (CABL, the South Australian state league); or Social: recreational competitions within metropolitan Adelaide, South Australia. Ethics approval was granted by the Human Research Ethics Committee of the University of South Australia (P162-05).

\subsection{Sample size}

An a priori power analysis run on the primary research question using $G^{*}$ Power v3.1 software (Heinrich Heine University of Düsseldorf, Düsseldorf, Germany) determined that a sample size of 180 was required to detect a medium effect size $(f)$ of 0.25 (Analysis of variance [ANOVA] with four groups: fixed effects, omnibus, one-way; based on Hönekopp and Schuster's, 2010 review) with an alpha of 0.05 and power of $80 \%$.

\subsection{Demographic information}

Participants were asked to complete a basic demographic and performance questionnaire. This questionnaire included data on age, height, mass, ethnicity, highest competitive standard achieved, playing team and positional data.

\subsection{D:4D measurement}

In this study, 2D:4D was measured using the photographic approach described by Hull et al. (2015), where digital measurements were extracted from photographs taken of each participant's hands. Briefly, participants were asked to place their hands flat on a table and directly underneath a tripod mounted Canon PowerShot A2000 IS digital camera (Canon, Tokyo, Japan), with the palmar surface facing up and fingers outstretched. Care was taken to ensure that the basal creases and the tips of the second and fourth digits of both hands were clearly visible. All digit photographs were imported into Adobe Photoshop (Adobe, San Jose, CA) to identify the XY coordinates corresponding to the basal creases and tips (at the midline) of the second and forth digits of both hands. Coordinate data were entered into Excel (Microsoft, Redmond, CA), with Cartesian coordinate geometry used to determine the length of each digit. Digit ratios for the right $\left(2 \mathrm{D}: 4 \mathrm{D}_{\mathrm{R}}\right)$ and left $\left(2 \mathrm{D}: 4 \mathrm{D}_{\mathrm{L}}\right)$ hands were calculated by dividing the length of the corresponding second digit and fourth digits. This method has demonstrated very good repeatability (Allaway et al., 2009; Hull et al., 2015; Ranson et al., 2013) and validity (vs. manual measurements) (Hull et al., 2015) and is an effective field-based measure to use with large populations. Furthermore, it does not require that fingers are placed downwards onto a glass surface as with indirect methods (e.g., photocopies or scans) of capturing images of fingers. Contact with the glass platen of a scanner or photocopier may distort finger tips and influence 2D:4D (Ribeiro et al., 2016).

Intra- and inter-tester repeatability were assessed using a subsample of 30 basketball players. Intra-tester repeatability was determined by comparing duplicate digital measurements of a single researcher (NF vs. $\mathrm{NF}$ ), with inter-tester repeatability determined by comparing single measurements of two researchers (NF vs. $\mathrm{MH}$ ). The 2D:4Ds demonstrated very good intra-and inter-tester repeatability, with negligible systematic errors (median change in means [95\% CI]: $-0.28 \%[-0.57,0.01]$ ), negligible-to-small random errors (median typical error [95\% CI]: $0.66 \%[0.60,0.72]$ ) and nearly perfect test-retest correlations (median intraclass correlation [95\% CI]: 0.98 [0.97, 0.99]). 


\subsection{Game-related statistics}

Game-related statistics were available for 57 players (48 national and 9 international level) from a single NBL season. Statistics were retrieved from the official website of the NBL (www.nbl.com.au). These game-related statistics were collected by professional NBL statisticians and published as public access official game statistics for the NBL and included: points scored, rebounds, assists, field goal percentage (FG\%), three point percentage (3FG\%), free throw percentage (FT\%), steals, blocks, turnovers and average minutes played. All individual game-related statistics were standardized to the metric of per 36 minutes (using the following formula) to remove the influence of playing time and number of games played.

$\frac{\text { Game-related Statistic }}{\text { Games Played } \times\left(\frac{36}{\text { Minutes per game }}\right)}$

\subsection{Statistical analysis}

One-way ANOVA was used to quantify the differences in mean $2 \mathrm{D}: 4 \mathrm{D}_{\mathrm{R}}$ and $2 \mathrm{D}: 4 \mathrm{D}_{\mathrm{L}}$ values across the four competitive standards (international, national, state and social). Effect sizes (eta squared $\left[\eta^{2}\right]$ ) for ANOVA of 0.01, 0.06, and 0.14 were used as thresholds for small, moderate and large (Cohen, 1988). An alpha level of 5\% was used as the criterion for significance, with probabilities corrected using sequential Bonferroni adjustments. Where Bonferroni corrected main effects were significant, post-hoc comparisons were made using Fisher's Partial Least Significant Difference to determine which between-group differences were statistically significant. Standardized two-group differences of $0.2,0.5$, and 0.8 were used as thresholds for small, moderate, and large, with differences $<0.2$ considered to be negligible and $\geq 0.2$ considered the "smallest important" difference (Cohen, 1988).

Linear relationships between the 2D:4Ds and basketball game-related statistics were quantified using Pearson's product moment correlation, with correlations of $0.1,0.3$, and 0.5 used as thresholds for weak, moderate, and strong (Cohen, 1988). All probabilities were corrected using sequential Bonferroni adjustments.

\section{Results}

Descriptive statistics for the final sample of Australian male basketball players are shown in Table $\underline{1}$. Participants represented four competitive standards: international $(n=25)$, national $(n=50)$, state $(n=88)$, and social $(n=58)$.

Table 1. Descriptive statistics (mean $\pm \mathrm{SD}$ ) for the included participants

\begin{tabular}{|l|l|l|l|l|l|l|}
\hline Group & $\boldsymbol{n}$ & Age (y) & $\begin{array}{l}\text { Height } \\
(\mathbf{m})\end{array}$ & Mass (kg) & 2D:4D $_{\mathbf{R}}$ & 2D:4D $_{\mathbf{L}}$ \\
\hline International & 25 & $28.5 \pm 5.3$ & $2.00 \pm 0.09$ & $100.4 \pm 12.1$ & $0.947 \pm 0.05$ & $0.939 \pm 0.05$ \\
\hline National & 50 & $27.1 \pm 5.1$ & $1.98 \pm 0.07$ & $97.7 \pm 10.1$ & $0.959 \pm 0.05$ & $0.953 \pm 0.05$ \\
\hline State & 88 & $22.1 \pm 3.2$ & $1.90 \pm 0.08$ & $86.6 \pm 10.0$ & $0.969 \pm 0.04$ & $0.967 \pm 0.04$ \\
\hline Social & 58 & $25.5 \pm 5.8$ & $1.84 \pm 0.08$ & $86.0 \pm 15.1$ & $0.962 \pm 0.03$ & $0.963 \pm 0.03$ \\
\hline
\end{tabular}

- $\quad 2 \mathrm{D}: 4 \mathrm{D}_{\mathrm{R}}=$ right $2 \mathrm{D}: 4 \mathrm{D}, 2 \mathrm{D}: 4 \mathrm{D}_{\mathrm{L}}=$ left $2 \mathrm{D}: 4 \mathrm{D}$.

\subsection{Differences in means between competitive standards}

One-way ANOVA revealed a statistically significant and small Bonferroni corrected main effect for $2 \mathrm{D}: 4 \mathrm{D}_{\mathrm{L}}$ and competitive standard $\left(F_{3,217}=3.6, P=0.01, \eta^{2}=0.05\right.$; Figure 1$)$. Post-hoc comparisons indicated that international players had statistically significant and moderately lower $2 \mathrm{D}: 4 \mathrm{D}_{\mathrm{L}}$ values than 
state (difference in means $[95 \% \mathrm{CI}]:-0.027[-0.045,-0.010]$ absolute units or $-0.70[-1.15,-0.24]$ standardized units) and social (difference in means: $-0.024[-0.041,-0.006]$ absolute units or -0.64 $[-1.12,-0.16]$ standardized units) players. Negligible-to-small and non-statistically significant differences were observed for all other between-group comparisons. There were no statistically significant main effect for $2 \mathrm{D}: 4 \mathrm{D}_{\mathrm{R}}\left(F_{3,217}=2.2, P=0.09, \eta^{2}=0.03\right)$.

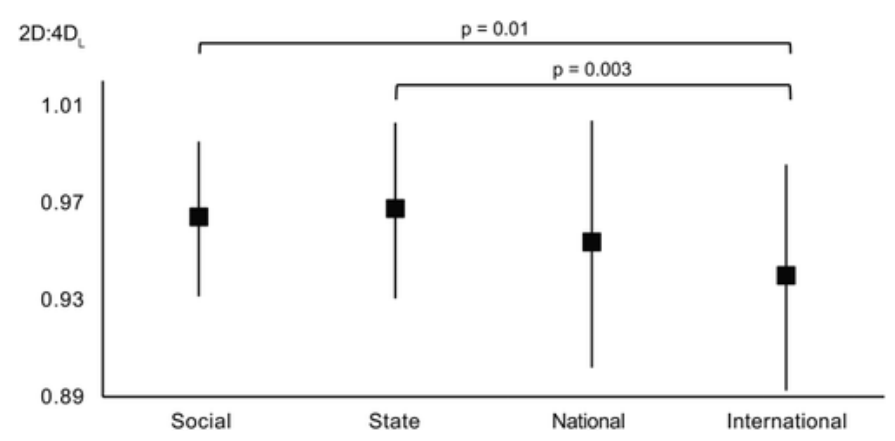

Figure 1

Means and standard deviations for $2 \mathrm{D}: 4 \mathrm{D}_{\mathrm{L}}$ across the four competitive standards. The probabilities are associated with post-hoc PLSD testing

\subsection{Correlations between 2D:4D and game-related statistics}

Correlations ranged from a weak, positive correlation between $2 \mathrm{D}: 4 \mathrm{D}_{\mathrm{R}}$ and free throw percentage (suggesting that men with higher 2D:4Ds were more accurate free throw shooters) to a weak, negative correlation between 2D:4D and assists (indicating that men with lower left 2D:4Ds delivered more assists) (Table 2). Overall, there were negligible-to-weak and non-statistically significant relationships between 2D:4D and basketball game-related statistics, with an overall mean $(95 \% \mathrm{CI})$ correlation of $-0.01(-0.07,0.05)$ (Table 2).

Table 2. Correlations ( $r$ values and the corresponding 95\% confidence limits) between 2D:4D and basketball game-related statistics

\begin{tabular}{|l|l|l|}
\hline Game-related statistic & 2D:4D & 2D:4D \\
\hline Points & $-0.19(-0.43,0.07)$ & $-0.14(-0.39,0.13)$ \\
\hline Assists & $0.08(-0.18,0.33)$ & $-0.25(-0.48,0.01)$ \\
\hline Rebounds & $-0.19(-0.43,0.07)$ & $0.05(-0.21,0.31)$ \\
\hline Blocks & $0.16(-0.10,0.40)$ & $0.01(-0.25,0.27)$ \\
\hline Steals & $0.02(-0.24,0.28)$ & $-0.03(-0.29,0.23)$ \\
\hline Turnovers & $0.18(-0.08,0.42)$ & $-0.01(-0.27,0.25)$ \\
\hline FG\% & $0.02(-0.24,0.28)$ & $-0.03(-0.29,0.23)$ \\
\hline 3FG\% & $0.10(-0.16,0.35)$ & $-0.14(-0.39,0.13)$ \\
\hline FT\% & $0.20(-0.06,0.44)$ & $-0.05(-0.31,0.21)$ \\
\hline
\end{tabular}

- Note: All statistics are per $36 \mathrm{~min}, \mathrm{FG} \%=$ field goal percentage, $3 \mathrm{FG} \%=$ three point field goal percentage and $\mathrm{FT} \%=$ free throw percentage. Because of the difference in direction of the turnovers statistic (where higher turnovers equate to poorer performance) the direction of the relationship between turnovers and 2D:4D was reversed, to ensure negative correlations between 2D:4Ds and gamerelated statistics indicated that lower 2D:4Ds were associated with better performance.

\section{Discussion}

This study found that $2 \mathrm{D}: 4 \mathrm{D}_{\mathrm{L}}$ significantly discriminated between Australian basketballers playing at different competitive standards, with moderate differences observed between international players (in the direction of lower 2D:4Ds for international players) and their state and social level counterparts. This finding is broadly consistent with previous literature (Hönekopp and Schuster, 2010) whereby a general trend of 2D:4Ds can be observed across groups (social $>$ state $>$ national $>$ international). However, $2 \mathrm{D}: 4 \mathrm{D}_{\mathrm{R}}$ did not significantly discriminate between competitive standards, although the main effect for 
2D:4D $\mathrm{D}_{\mathrm{R}}$ trended towards significance $(P=0.09)$. Despite the $2 \mathrm{D}: 4 \mathrm{D}$ being a nonfunctional trait, there is mounting evidence suggesting the 2D:4D may reflect the organizational and activational benefits of testosterone which may explain why a lower $2 \mathrm{D}: 4 \mathrm{D}_{\mathrm{L}}$ is observed for basketball players reaching higher competitive standards.

The other main finding from this study was that 2D:4D was not meaningfully related to basketball gamerelated statistics among national-level players, which is not entirely consistent with previous research showing overall weak-to-moderate and negative correlations between 2D:4Ds and sporting/physical performance (Hönekopp and Schuster, 2010). This finding may be somewhat explained by the differences between closed- and open-skill sports. Performance in open-skill sports (or open-skill dominant sports such as basketball) is not usually meaningfully related to single physical or physiological measures (such as 2D:4D) because of the multitude of factors involved in success (determined by collective actions of each player, not one individual; Jonker et al., 2010). The Burgess and Naughton (2010) review provides evidence for this, finding that while physiological and physical measures of performance translate well in closed-skill sports, the application to open-skill sports is, however, unclear. Another issue to consider is sample heterogeneity, which is known to affect correlation estimates. The nature of selection for representative/elite sport is based on a range of strict physical, physiological, skill, and behavioral criteria which often act to decrease performance variability (a survival of the "fittest" if you will). This range restriction in performance can lead to weaker correlations being observed, which may in part explain why negligible-to-weak correlations were observed at the national-level.

This study has several strengths. First, this study adds to the paucity of literature relating 2D:4Ds to physical performance in team sports, especially court-based team sports. Second, while manual 2D:4D data are widely collected, this study used a photographic technique and Cartesian coordinate geometry to determine digit lengths and 2D:4Ds that demonstrated very good repeatability and has previously demonstrated very good agreement with manual measurements (Hull et al., 2015). This protocol also avoids the potential confound of placing fingers downwards onto a glass platen of a scanner or photocopier. Thus it avoids possible distortion of the fat pads of the finger tips (Ribeiro et al., 2016). Third, it used a strict set of inclusion/exclusion criteria to eliminate potential sources of bias (i.e., the analysis was restricted to men of European descent because of known ethnic differences in 2D:4Ds). Nonetheless, this study is not without its limitations. Every effort was made to capture a large, representative sample of players at each competitive standard, although this proved difficult for the state, national and international players who were in competition at the time of testing. For example, state players were tested during in-season team practices, national players during a pre-season tournament, and many of the international players (who were based overseas) during Australian national team camps. We also classified players based on their highest competitive standard at the time of testing, and it is possible that some of the younger national players ultimately competed internationally (i.e., they subsequently represented Australia at the Olympics and/or World Cup). Another limitation of this study is that no additional information was available on the non-basketball athletic achievements of the social players, and it is possible that some played at higher competitive standards in other sports (e.g., at the state and national levels), resulting in 2D:4Ds that may be lower than that of true social-level players.

This study suggests that 2D:4D may be a discriminator of basketball performance between, but not

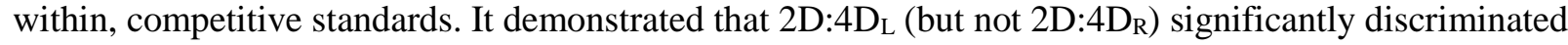
between Australian men playing at different competitive standards of basketball, with none of the 2D:4Ds meaningfully relating to basketball game-related statistics among national-level players. Further investigations should focus on relationships between the 2D:4D and team sports performance, especially court-based sports, both within and between competitive standards. 


\section{Acknowledgments}

The authors thank Mr. Nikola Popovic for his help collecting digit ratio data on national and international players.

\section{Author Contributions}

$\mathrm{NF}, \mathrm{MH}$, and GT designed the study, GT collected the data. NF, MH, JM, and GT analyzed the data and drafted the manuscript.

\section{References}

Allaway, H. C., Bloski, T. G., Pierson, R. A., \& Lujan, M. E. (2009). Digit ratios (2D:4D) determined by computer-assisted analysis are more reliable than those using physical measurements, photocopies, and printed scans. American Journal of Human Biology, 21(3), 365-370.

Burgess, D. J., \& Naughton, G. A. (2010). Talent development in adolescent team sports: A review. International Journal of Sports Physiology and Performamce, 5(1), 103-116.

Cohen, J. (1988). Statistical power analysis for the behavioral sciences. Hillsdale, New Jersery: Lawrence Erlbaum. Geschwind, N., \& Galaburda, A. (1987). Cerebral lateralization. Cambridge, Massachusetts: MIT Press.

H€onekopp, J., \& Schuster, M. (2010). A meta-analysis on 2D:4D and athletic prowess: Substantial relationships but neither hand out-predicts the other. Personality and Individual Differences, 48(1), 4-10.

Hull, M. J., Schranz, N. K., Manning, J. T., \& Tomkinson, G.R. (2015). Relationships between digit ratio (2D: 4D) and female competitive rowing performance. American Journal of Human Biology, $27(2), 157-163$.

Jonker, L., Elferink-Gemser, M. T., \& Visscher, C. (2010).Differences in self-regulatory skills among talented athletes: The significance of competitive level and type of sport. Journal of Sports Science, 28(8), 901-908.

Kimura, D. (1996). Sex, sexual orientation and sex hormones influence human cognitive function. Current Opinion in Neurobiology, 6(2), 259-263.

Malas, M. A., Dogan, S., Evcil, E. H., \& Desdicioglu, K. (2006). Fetal development of the hand, digits and digit ratio (2D: 4D). Early Human Development, 82(7), 469-475.

Mann, D. T., Williams, A. M., Ward, P., \& Janelle, C. M. (2007). Perceptual-cognitive expertise in sport: A meta-analysis. Journal of Sport and Exercise Psychology, 29(4), 457.

Manning, J. T. (2002). Digit ratio: A pointer to fertility, behaviour and health. New Jersey: Rutgers University Press.

Manning, J. T., Churchill, A. J., \& Peters, M. (2007). The effects of sex, ethnicity, and sexual orientation on self-measured digit ratio (2D: 4D). Archives of Sexual Behavior, 36(2), 223-233.

Manning, J. T., \& Hill, M. R. (2009). Digit ratio (2D:4D) and sprinting speed in boys. American Journal of Human Biology, 21(2), 210-213. 
Manning, J. T., \& Taylor, R. P. (2001). Second to fourth digit ratio and male ability in sport: Implications for sexual selection in humans. Evolution and Human Behavior, 22(1), 61-69.

McIntyre, M. H., Ellison, P. T., Lieberman, D. E., Demerath, E., \& Towne, B. (2005). The development of sex differences in digital formula from infancy in the Fels Longitudinal Study. Proceeding of Royal Society London [Biol], 272(1571), 1473-1479.

Mortlock, D. P., \& Innis, J. W. (1997). Mutation of HOXA13in hand-foot-genital syndrome. Nature Genetics, 15(2), 179-180.

Panda, K., Majumdar, P., Umesh, V., \& Sudhakar, H. (2014). Ratio of second to fourth digit as a predictor of performance in elite Indian volley ball players. National Journal of Physiology Pharmacy and Pharmacology, 4(2), 106-108.

Peters, M., Manning, J. T., \& Reimers, S. (2007). The effects of sex, sexual orientation, and digit ratio (2D: 4D) on mental rotation performance. Archives of Sexual Behavior, 36(2), 251-260.

Ranson, R., Taylor, S., \& Stratton, G. (2013). Reliability of afield based 2D: 4D measurement technique in children. Early Human Development, 89(8), 589-592.

Ribeiro, E., Neave, N., Morais, R. N., \& Manning, J. T. (2016). Direct versus indirect measurement of digit ratio (2D: 4D) a critical review of the literature and new data. Evolutionary Psychology, 14(1), doi:10.1177/1474704916632536.

Schorer, J., Rienhoff, R., Westphal, H., \& Baker, J. (2013). Digit ratio effects between expertise levels in American football players. Talent Development and Excellence, 5(2),113-116.

Sudhakar, H. H., Majumdar, P., Umesh, V., \& Panda, K.(2014). Second to fourth digit ratio is a predictor of sporting ability in elite Indian male kabaddi players. Asian Journal of Sports Medicine, 5(3), e23073.

Taddei, F., Bultrini, A., Spinelli, D., \& Di Russo, F. (2012). Neural correlates of attentional and executive processing in middle-age fencers. Medicine and Science in Sports and Exercise, 44(6), 10571066.

Tester, N., \& Campbell, A. (2007). Sporting achievement: What is the contribution of digit ratio? Journal of Personality, 75(4), 663-677.

Tlauka, M., Williams, J., \& Williamson, P. (2008). Spatial ability in secondary school students: Intra-sex differences based on self-selection for physical education. British Journal of Psychology, 99(Part 3), 427-440.

Trivers, R., Manning, J., \& Jacobson, A. (2006). A longitudinal study of digit ratio (2D: 4D) and other finger ratios in Jamaican children. Hormones and Behavrior, 49(2), 150-156.

Voss, M. W., Kramer, A. F., Basak, C., Prakash, R. S., \&Roberts, B. (2010). Are expert athletes "expert" in the cognitive laboratory? A meta-analytic review of cognition and sport expertise. Applied Cognitive Psychology, 24(6), 812-826.

Wang, C.-H., Chang, C.-C., Liang, Y.-M., Shih, C.-M., Chiu, W.-S., Tseng, P.,...Juan, C.-H. (2013). Open vs. closed skill sports and the modulation of inhibitory control. PloSOne, 8(2), e55773. 
Zheng, Z., \& Cohn, M. J. (2011). Developmental basis of sexually dimorphic digit ratios. Proceedings of National Academy of Science of the United States of America, 108(39),16289-16294. 